\title{
Proton decay in product group unification
}

\author{
Jason L. Evans, ${ }^{1}$ Masahiro Ibe, ${ }^{2,3}$ and Tsutomu T. Yanagida ${ }^{1,3}$ \\ ${ }^{1}$ T. D. Lee Institute, Shanghai 200240, China \\ ${ }^{2}$ Institute for Cosmic Ray Research (ICRR), The University of Tokyo, Chiba 277-8583, Japan \\ ${ }^{3}$ Kavli Institute for the Physics and Mathematics of the Universe (WPI), The University of Tokyo Institutes \\ for Advanced Study, The University of Tokyo, Kashiwa 277-8583, Japan
}

(Received 3 November 2020; accepted 21 December 2020; published 9 February 2021)

\begin{abstract}
Product group unification is an attractive alternative to simple grand unification. It solves the infamous doublet-triplet splitting problem and the dimension-5 proton decay problems without introducing any finetuning. Furthermore, the matter multiplets are still embedded into unified SU(5) representations. In this paper, we discuss proton decay for the simplest product group unification model based on $\mathrm{SU}(5) \times \mathrm{U}(2)_{\mathrm{H}}$. We find that the minimal setup of the model has already been excluded by dimension- 6 proton decay. We also show that a simple extension of the model, with naturally generated SU(5) incomplete multiplets, can rectify this problem. We find that the proton lifetime will be in reach of coming experiments like DUNE and Hyper-K, when the mass of the incomplete multiplet is associated with the Peccei-Quinn-symmetry breaking. In this case, the dark matter may be an admixture of the Wino lightest supersymmetric particle and the axion.
\end{abstract}

DOI: 10.1103/PhysRevD.103.035009

\section{INTRODUCTION}

One of the challenges of supersymmetric (SUSY) grand unified theories (GUTs) is the doublet-triplet splitting. In the minimal model of SU(5), for example, the Higgs bosons must be embedded in a $\mathbf{5}, \overline{\mathbf{5}}$. This means that the doublet Higgs bosons are accompanied by SU(3) triplets. The presence of a triplet Higgs boson, at the energy scale of the minimal supersymmetric standard model (MSSM), ruins the precise coupling unification at the GUT scale. In addition to this complication, the predicted lifetime for the proton through the exchange of this very light triplet Higgs boson would be in conflict with experimental constraints. Thus, the doublet-triplet splitting is required. In minimal SU(5), this splitting is accomplished by a severe fine-tuning.

The minimal SU(5) model is further complicated by the fact that the proton, lifetime in the channel $p \rightarrow K^{+} \bar{\nu}$, tends to be too short unless the soft masses are quite large and the phases in the Yukawa couplings are chosen appropriately ${ }^{1}$

\footnotetext{
${ }^{1}$ Minimal SU(5) models are further complicated by the fact that the operator $\overline{\mathbf{5 1 0 1 0 1 0}} / M_{P}$ is allowed by all the symmetries. Unless the coefficient of this operator is quite small, the short proton lifetime rules out all low-scale SUSY models.

Published by the American Physical Society under the terms of the Creative Commons Attribution 4.0 International license. Further distribution of this work must maintain attribution to the author(s) and the published article's title, journal citation, and DOI. Funded by SCOAP ${ }^{3}$.
}

[1,2] (for recent work, see e.g., [3-5]). This has led to the study of more sophisticated model.

Several solutions to the doublet-triplet splitting have been proposed. One candidate, missing partner models $[6,7]$, use a $\mathbf{7 5}$ to break $\mathrm{SU}(5)$ down to the standard model (SM) gauge symmetries. In this case, the Higgs bosons, which reside in a $\mathbf{5}, \overline{\mathbf{5}}$, are coupled a $\mathbf{5 0}, \overline{\mathbf{5 0}}$ through the $\mathbf{7 5}$. Since the 50, $\overline{\mathbf{5 0}}$ do not contain any doublets, the Higgs bosons bilinear mass is not generated. Although this model solves the doublet-triplet splitting problem, a more complicated structure is needed to forbid the $\mathbf{5} \overline{\mathbf{5}}$ Higgs bilinear term. This structure tends to be plagued by other problems. Other types of unification models, like flipped SU(5), also rely on a missing partner type mechanism to suppress the Higgs doublet mass. However, the $\mathbf{5} \overline{\mathbf{5}}$ Higgs bilinear mass term is set to zero by hand. ${ }^{2}$ Furthermore, models like flipped SU(5) completely lose the explanation of charge quantization.

In this paper, we will examine product group unification with the gauge symmetries $\mathrm{SU}(5) \times \mathrm{U}(2)_{\mathrm{H}}[9,10]$ (see Refs. [10-14] for the earlier works on this type of the product group unification models.). This product group unification model is characterized by having an R-symmetry which forbids the Higgs bilinear term $\mathbf{5} \overline{\mathbf{5}}$ and the dimension-5 proton decay operators simultaneously. ${ }^{3}$

\footnotetext{
${ }^{2}$ It is possible using R-symmetries to forbid the Higgs bilinear mass term in flipped $\mathrm{SU}(5)$; see [8].

${ }^{3}$ This also forbids the operator $\mathbf{5 1 0 1 0 1 0} / M_{P}$.
} 
For this model, the doublet-triplet splitting is accomplished without any unnaturally small couplings.

Furthermore, this model maintains the same matter field embeddings as minimal $\mathrm{SU}(5)$, that is, the standard model fields are contained in the $\mathbf{1 0}$ and $\overline{\mathbf{5}}$ just as in minimal $\mathrm{SU}(5)$. Although the $\mathrm{SM}$ gauge symmetries $\mathrm{SU}(2) \times \mathrm{U}(1)$ are the diagonal subgroup of $\mathrm{SU}(5) \times \mathrm{U}(2)_{\mathrm{H}}$, this embedding leads to a perceived charge quantization among the MSSM fields due to the embedding of SM fields in $\mathbf{1 0}$ and $\overline{\mathbf{5}}^{4}$

As we will see, the minimal $\mathrm{SU}(5) \times \mathrm{U}(2)_{\mathrm{H}}$ unification model has already been excluded by dimension- 6 proton decay experiments ${ }^{5}$ [15]. We also show that a simple extension of the model including new pairs of $\mathbf{5}, \overline{\mathbf{5}}$ and $\mathbf{2}, \overline{\mathbf{2}}$ can rectify this problem. In this extension, the mass of the doublets and the triplets embedded in the new $\mathbf{5 , 5}$ is split with the triplets being much lighter. These light triplets deflect the running of the gauge couplings and alter the gauge matching conditions. This leads to a larger mass for the heavy gauge bosons of $\mathrm{SU}(5)$ and a longer proton lifetime. As we will show, these light triplets can also be the heavy quarks which couple to the Peccei-Quinn (PQ) breaking field of the -Kim-Shifman-Vainshtein-Zakharov (KSVZ) axion scenario [16,17]. To push the proton lifetime beyond the current experimental limit, the triplet masses need to be smaller than about $10^{12} \mathrm{GeV}$. Suggestively, this scale implies that the axion makes up some portion of the dark matter. If the axion does indeed make of some nontrivial portion of the dark matter and we make some rather mild assumptions about order one couplings, the proton lifetime of this model will be in reach of coming experiments like DUNE [18] and Hyper-K [19] no matter the MSSM soft mass spectrum. Since dimension-6 proton decay dominates, this makes for a unique proton decay signature for these experiments to search for.

The paper is organized as follow. Section II gives a brief review of $\mathrm{SU}(5) \times \mathrm{U}(2)_{\mathrm{H}}$ production group unification. Next, in Sec. III, we discuss our calculation of the proton lifetime including discussion of the SUSY breaking scenario we use. In Sec. IV, we present our product group unification model, including some discussion on how the axion ties in to this scenario. Then, Sec. V presents the result of our proton lifetime calculation for the particular product group unification model we consider.

\section{THE MODEL}

The model we consider is based on that found in $[9,10]$ and is a unification model with the gauge symmetries $\mathrm{SU}(5) \times \mathrm{U}(2)_{\mathrm{H}}$. These symmetries are broken down to

\footnotetext{
${ }^{4}$ However, any charge is possible if the $\mathbf{1 0}$ 's and $\overline{\mathbf{5}}$ 's are initially charged under the $\mathrm{U}(1)_{\mathbf{H}}$ or there are other fields charged only under the $\mathrm{U}(2)_{\mathrm{H}}$ gauge symmetries.

${ }^{5}$ This is due the gauge coupling matching conditions requiring the $\mathrm{SU}(5)$ guage bosons to be light.
}

TABLE I. Charge assignments for all fields. We normalize the $\mathrm{U}(1)_{\mathrm{H}}$ charge so that the charge matrix on the $\mathrm{SU}(2)_{\mathrm{H}}$ doublet is $\left(\tau_{0}\right)_{\alpha \beta}=\delta_{\alpha \beta} / 2$.

\begin{tabular}{|c|c|c|c|c|c|c|c|c|c|c|c|c|c|}
\hline Fields & $\bar{\Phi}_{i}$ & $\Psi_{i}$ & & & $Q_{6}$ & $\bar{Q}_{6}$ & $Q$ & $\bar{Q}$ & $\Phi^{\prime}$ & $\bar{\Phi}^{\prime}$ & $\Theta$ & $\bar{\Theta}$ & $P$ \\
\hline $\mathrm{U}(5)$ & $\overline{5}$ & 10 & & 1 & 1 & 1 & $\overline{5}$ & 5 & 5 & $\overline{5}$ & 1 & 1 & 1 \\
\hline $\mathrm{SU}(2)_{\mathrm{H}}$ & 1 & 1 & 3 & 1 & 2 & 2 & 2 & 2 & 1 & 1 & 2 & 2 & 1 \\
\hline $\mathrm{U}(1)_{\mathrm{H}}$ & 0 & 0 & 0 & 0 & $-1 / 2$ & $1 / 2$ & $-1 / 2$ & $21 / 2$ & 0 & 0 & $-1 / 2$ & $21 / 2$ & 0 \\
\hline$R$ charge & 1 & 1 & 2 & 2 & 0 & 0 & 0 & 0 & 1 & 1 & 1 & 1 & 0 \\
\hline PQ charge & 0 & 0 & 0 & 0 & 0 & 0 & 0 & 0 & 1 & 0 & 0 & -1 & -1 \\
\hline
\end{tabular}

the $\mathrm{SM}$ gauge symmetries $\mathrm{SU}(3) \times \mathrm{SU}(2) \times \mathrm{U}(1)$ by the following superpotential:

$W=\sqrt{2} \lambda_{2 H} \bar{Q} X^{a} \tau^{a} Q+\sqrt{2} \lambda_{1 H} \bar{Q} X_{0} Q-\sqrt{2} \lambda_{1 H} v^{2} X_{0}$,

where we have suppressed gauge indices and the charge assignments are in Table I. The $\tau^{a}(a=1,2,3)$ denote half of the Pauli matrices, $v$ is the mass parameter of the GUT scale, while $\lambda$ 's are coupling constants. We follow the normalization of the coupling constants in [9]. The theory also has a well-defined R-symmetry as seen in Table I.

The theory is broken to the SM gauge symmetries by the vacuum expectation value (VEV),

$$
Q_{\alpha}^{A}=v \delta_{\alpha}^{A} \quad Q_{A}^{\alpha}=v \delta_{A}^{\alpha},
$$

where $\alpha, \beta \ldots$ are for the $\mathrm{SU}(2)$ indices and $A, B$.. refer to the SU(5) indices. After the gauge symmetry is broken, the masses of the particles are

$M_{X^{\prime}}=\sqrt{2} \lambda_{2 H} v \quad M_{Q_{\beta}^{\alpha}+\bar{Q}_{\beta}^{\alpha}}=\sqrt{2} \lambda_{2 H} v \quad M_{X_{0}}=\sqrt{2} \lambda_{1 H} v$,

where $M_{Q_{\beta}^{\alpha}+\bar{Q}_{\beta}^{\alpha}}$ is the mass of the linear combination $Q_{\beta}^{\alpha}+\bar{Q}_{\beta}^{\alpha}$ and the others we hope are self-explanatory. The orthogonal component $\bar{Q}_{\beta}^{\alpha}-Q_{\beta}^{\alpha}$ is one of the Goldstone boson fields associated with the breaking of the gauge symmetries. The other component of $Q, \bar{Q}$, involving $\mathrm{SU}(3)$ portion of the $\mathrm{SU}(5)$ indices, are also would-be Goldstone bosons. The gauge boson masses corresponding to the broken generators are as follows:

$$
\begin{aligned}
M_{X} & =g_{5} v, \quad M_{V_{\mathrm{U}(1)}}=\sqrt{2} \sqrt{g_{1 H}^{2}+\frac{3}{5} g_{5}^{2} v}, \\
M_{V_{\mathrm{SU}(2)}} & =\sqrt{2} \sqrt{g_{2 H}^{2}+g_{5}^{2}} v .
\end{aligned}
$$

It should be noted that the $\mathrm{SU}(5) \times \mathrm{U}(2)_{\mathrm{H}}$ breaking sector leaves no massless particles.

In this theory, we break the $\mathrm{SU}(2) \times \mathrm{U}(1)$ subgroup of $\mathrm{SU}(5)$ diagonally with the $\mathrm{U}(2)_{\mathrm{H}}$. This means that after the breaking, the $\mathrm{U}(2)_{\mathrm{H}}$ fields now have $\mathrm{SU}(2)_{\mathrm{W}} \times \mathrm{U}(1)_{\mathrm{Y}}$ charges. The charges of the massive gauge bosons are, in 
the notation $(\mathrm{SU}(3), \mathrm{SU}(2))_{\mathrm{U}(1)_{\mathrm{Y}}}, X(\mathbf{3}, \mathbf{2})_{5 / 3}, \bar{X}(\overline{\mathbf{3}}, \mathbf{2})_{-5 / 3}$, $V_{\mathrm{SU}(2)}(\mathbf{1}, \mathbf{3})_{0}$, and $V_{\mathrm{U}(1)}(\mathbf{1}, \mathbf{1})_{0}$. Using these charge assignments, we get the following matching conditions for the gauge couplings:

$$
\begin{gathered}
\frac{1}{g_{3}^{2}\left(M_{G}\right)}=\frac{1}{g_{5}^{2}\left(M_{G}\right)}+\frac{1}{2 \pi^{2}} \ln \left(\frac{M_{X}}{M_{G}}\right), \\
\frac{1}{g_{2}^{2}\left(M_{G}\right)}=\frac{1}{g_{5}^{2}\left(M_{G}\right)}+\frac{1}{g_{2 H}^{2}}+\frac{3}{4 \pi^{2}} \ln \left(\frac{M_{X}}{M_{G}}\right) \\
+\frac{1}{2 \pi^{2}} \ln \left(\frac{M_{V_{\mathrm{SU}(2)}}}{M_{Q_{\beta}^{\alpha}+\bar{Q}_{\beta}^{\alpha}}^{\alpha}}\right), \\
\frac{1}{g_{1}^{2}\left(M_{G}\right)}=\frac{1}{g_{5}^{2}\left(M_{G}\right)}+\frac{3}{5} \frac{1}{g_{1 H}^{2}}+\frac{5}{4 \pi^{2}} \ln \left(\frac{M_{X}}{M_{G}}\right),
\end{gathered}
$$

where we use $M_{G}$ to indicate the matching scale. When we implement these matching conditions, we will use the scale at which $g_{1}=g_{2}$. From Eqs. (5) and (7), we can find $M_{X}$ in terms of $g_{1 H}^{2}$,

$M_{X}=M_{G} \exp \left(\frac{4 \pi^{2}}{3}\left[\frac{1}{g_{1}^{2}\left(M_{G}\right)}-\frac{1}{g_{3}^{2}\left(M_{G}\right)}-\frac{3}{5} \frac{1}{g_{1 H}^{2}\left(M_{G}\right)}\right]\right)$.

Since the couplings unify quite well in supersymmetry, the differences of the MSSM gauge couplings are quite small. If $g_{1 H}^{2}$ is of order $4 \pi$, then $M_{X}$ is quite close to the unification scale. However, if $g_{1 H}^{2}$ is of order one, then $M_{X}$ is much lower than the unification scale. As we will see, this leads to a proton lifetime which is in conflict with experimental constraints.

The gauge coupling $g_{5}$, which is important for proton decay, can be found from Eq. (5),

$$
g_{5}\left(M_{G}\right)=\left[\frac{1}{g_{3}^{2}\left(M_{G}\right)}-\frac{1}{2 \pi} \ln \left(\frac{M_{X}}{M_{G}}\right)\right]^{-\frac{1}{2}} .
$$

Since $M_{X}$ is known in terms of $g_{1 H}$, both $g_{5}$ and $M_{X}$ are determined by choosing $g_{1 H}$.

\section{A. MSSM Yukawa couplings}

As a notable feature of the $\mathrm{SU}(5) \times \mathrm{U}(2)_{\mathrm{H}}$ unification model, there are no $\mathbf{5}$ and $\overline{\mathbf{5}}$ Higgs bosons. Instead, the Higgs bosons arise from additional massless fields charged under only $\mathrm{U}(2)_{\mathrm{H}}, Q_{6}$, and $\bar{Q}_{6}$ in Table I. ${ }^{6}$ Since these fields will be charged under the $\mathrm{SM} \mathrm{SU}(2) \times \mathrm{U}(1)_{\mathrm{Y}}$ once the $\mathrm{SU}(5) \times \mathrm{U}(2)$ breaks to the $\mathrm{SM}$ gauge symmetries, these fields can play the role of Higgs boson. At the tree level, the

\footnotetext{
${ }^{6}$ The $Q_{6}$ and $\bar{Q}_{6}$ are nothing but the pseudo-Nambu-Goldstone chiral multiplets in the limit of $\lambda_{2 H}=\lambda_{1 H}$ [14].
}

Higgs bosons cannot interact with the MSSM matter content. The only allowed tree-level interactions of the Higgs bosons are

$$
W_{H}=\sqrt{2} \lambda_{2 H}^{\prime} \bar{Q}_{6} X Q_{6}+\sqrt{2} \lambda_{1 H}^{\prime} \bar{Q}_{6} X_{0} Q_{6}
$$

In the above expression, there is no supersymmetric mass for the $Q_{6}, \bar{Q}_{6}$ since their R-charge is zero. As there is no triplet Higgs, the model is free from the doublet-triplet splitting problem by construction. We will return to the generation of the Higgs supersymmetric bilinear mass later.

The MSSM Yukawa couplings are generated from higher dimensional operators,

$$
W_{Y}=\frac{c_{5_{i j}} Q \bar{Q}_{6}}{\Lambda} \Psi_{i} \bar{\Phi}_{j}+\frac{c_{10_{i j}} \bar{Q} Q_{6}}{\Lambda} \Psi_{i} \Psi_{j},
$$

where $\Lambda$ is the cutoff of the theory. The $\bar{\Phi}$ and $\Psi$ above contain all the MSSM fields with $i, j$ being the flavor indices (see Table I). They both also have an R-charge of 1 . To reproduce the top Yukawa coupling in the MSSM, we require that the cutoff scale $\Lambda$ is not far from $\mathcal{O}(\langle Q\rangle)$ but larger.

Now the expressions for the Yukawa couplings require a little more careful treatment, since the $\mathrm{SU}(2)_{\mathrm{H}}$ and $\mathrm{U}(1)_{\mathrm{H}}$ are not asymptotically free. In this case, we identify $\Lambda$ with the confinement scale of some strong interacting ultraviolet (UV) theory. Unless the Landau-pole scale is separated from the GUT scale, $\mathcal{O}(v)$, by at least an order of magnitude, the expressions for the Yukawa couplings above are not well defined.

This needed separation of scales has implications for the gauge couplings. If we enforce $\Lambda \gtrsim 4 \pi\langle Q\rangle$, the gauge couplings for $\mathrm{SU}(2)_{\mathrm{H}}$ and $\mathrm{U}(1)_{\mathrm{H}}$ will be suppressed at the GUT scale due to the renormalization group (RG) running. That is, even if we set them equal to $4 \pi$ at the cutoff scale $\Lambda$, they will no longer be of order $4 \pi$ at the GUT scale. This RG running will place an upper limit on the size of the $g_{1 H}$ and $g_{2 H}$ at the GUT scale which is less than $4 \pi$. This will in turn affect the upper bound on the mass of $M_{X}$, as seen in Eq. (8). Since $M_{X}$ only depends on $g_{1 H}$, we will focus on the RG effects on this coupling. We will only consider the one-loop renormalization group equations (RGEs), using them as a guide. The one-loop RGE for $g_{1 H}$ is

$$
\frac{d g_{1 H}^{2}}{d \ln \mu}=6 \frac{g_{1 H}^{4}}{8 \pi^{2}}
$$

The solution to these one-loop equations is

\footnotetext{
${ }^{7}$ The top Yukawa couplings for the models we consider below are of order $0.45-0.6$ depending on the value of $\tan \beta$ and the Higgs soft masses, which require a rather large coupling $c_{10}$.
} 


$$
g_{1 H}^{2}(\mu)=\frac{g_{1 H}^{2}(\Lambda)}{1-6 \frac{g_{1 H}^{2}(\Lambda)}{8 \pi^{2}} \ln \left(\frac{\mu}{\Lambda}\right)}
$$

Using this equation, we can determine the maximum size of the coupling that allows a $4 \pi$ separation between the Landau pole, $\Lambda$ and GUT scale, which we take to be $M_{G}$ throughout the rest of this work. This is roughly estimated by taking $g_{1 H}^{2}(\Lambda)=\Lambda / \mu=4 \pi$ in the above equation, which gives

$$
g_{1 H}^{2}(\langle Q\rangle)=g_{1 H}^{2}(\Lambda / 4 \pi)=3.68 .
$$

As we will see below, a value this small leads to a proton lifetime which is much too short in the minimal $\mathrm{SU}(5) \times \mathrm{U}(2)_{\mathrm{H}}$ model we have discussed above.

\section{PROTON DECAY}

In product group unification, there is no dimension-5 proton decay, since the operator $\mathbf{5 1 0 1 0 1 0}$ is forbidden by the R-symmetry. This is already a significant deviation from minimal SU(5) where this is the dominant decay mode.

Dimension-6 proton decay, on the other hand, proceeds as usual. Here we will give some details of the dimension-6 proton decay calculation. The important interactions for dimension-6 proton decay are

$\mathcal{L}_{\text {int }}=\frac{g_{5}}{\sqrt{2}}\left[-\overline{d_{R i}^{c}} X L_{i}+e^{-i \varphi_{i}} \bar{Q}_{i} X u_{R i}^{c}+\overline{e_{R i}^{c}} X\left(V^{\dagger}\right)_{i j} Q_{j}+\right.$ H.c. $]$,

where $V_{i j}$ are the Cabibbo-Kobayashi-Maskawa matrix elements. To calculate the proton lifetime induced by these operators, we first integrate out the $X$ boson. We then evolve these operators Wilson's coefficients down to the hadronic scale using renormalization group equations, making the necessary adjustments to the equations at the SUSY and weak scale. The decay width is then calculated at the hadronic scale ${ }^{8}$ for different leptons flavors $\ell_{i}$,

$$
\begin{aligned}
\Gamma\left(p \rightarrow \pi^{0} \ell_{i}^{+}\right)= & \frac{m_{p}}{32 \pi}\left(1-\frac{m_{\pi}^{2}}{m_{p}^{2}}\right)^{2}\left[\left|\mathcal{A}_{L}\left(p \rightarrow \pi^{0} \ell_{i}^{+}\right)\right|^{2}\right. \\
& \left.+\left|\mathcal{A}_{R}\left(p \rightarrow \pi^{0} \ell_{i}^{+}\right)\right|^{2}\right],
\end{aligned}
$$

where $m_{p}$ and $m_{\pi}$ are the proton and pion masses, respectively. The amplitudes are given by

\footnotetext{
${ }^{8}$ We take the bottom quark mass as the hadronic scale.
}

$$
\begin{aligned}
& \mathcal{A}_{L}\left(p \rightarrow \pi^{0} \ell_{i}^{+}\right)=-\frac{g_{5}^{2}}{M_{X}^{2}} \delta_{i 1} \cdot A_{1} \cdot\left\langle\pi^{0}\left|(u d)_{R} u_{L}\right| p\right\rangle_{i}, \\
& \mathcal{A}_{R}\left(p \rightarrow \pi^{0} \ell_{i}^{+}\right)=-\frac{g_{5}^{2}}{M_{X}^{2}}\left(\delta_{i 1}+V_{u d} V_{u i}^{*}\right) \cdot A_{2} \cdot\left\langle\pi^{0}\left|(u d)_{L} u_{R}\right| p\right\rangle_{i},
\end{aligned}
$$

where $A_{1,2}$ take care of the RG running, and $\left\langle\pi^{0}\left|(u d)_{(R, L)} u_{(L, R)}\right| p\right\rangle_{i}$ are the hadron matrix elements for decays to $\ell_{i}$. The RG coefficients $A_{1,2}$ are given by

$$
\begin{aligned}
A_{1}= & A_{L} \cdot\left[\frac{\alpha_{3}\left(M_{\mathrm{SUSY}}\right)}{\alpha_{3}\left(M_{\mathrm{GUT}}\right)}\right]^{\frac{4}{9}}\left[\frac{\alpha_{2}\left(M_{\mathrm{SUSY}}\right)}{\alpha_{2}\left(M_{\mathrm{GUT}}\right)}\right]^{-\frac{3}{2}}\left[\frac{\alpha_{1}\left(M_{\mathrm{SUSY}}\right)}{\alpha_{1}\left(M_{\mathrm{GUT}}\right)}\right]^{-\frac{1}{18}} \\
& \times\left[\frac{\alpha_{3}\left(m_{Z}\right)}{\alpha_{3}\left(M_{\mathrm{SUSY}}\right)}\right]^{\frac{2}{7}}\left[\frac{\alpha_{2}\left(m_{Z}\right)}{\alpha_{2}\left(M_{\mathrm{SUSY}}\right)}\right]^{\frac{27}{38}}\left[\frac{\alpha_{1}\left(m_{Z}\right)}{\alpha_{1}\left(M_{\mathrm{SUSY}}\right)}\right]^{-\frac{11}{82}}, \\
A_{2}= & A_{L} \cdot\left[\frac{\alpha_{3}\left(M_{\mathrm{SUSY}}\right)}{\alpha_{3}\left(M_{\mathrm{GUT}}\right)}\right]^{\frac{4}{9}}\left[\frac{\alpha_{2}\left(M_{\mathrm{SUSY}}\right)}{\alpha_{2}\left(M_{\mathrm{GUT}}\right)}\right]^{-\frac{3}{2}}\left[\frac{\alpha_{1}\left(M_{\mathrm{SUSY}}\right)}{\alpha_{1}\left(M_{\mathrm{GUT}}\right)}\right]^{-\frac{23}{198}} \\
& \times\left[\frac{\alpha_{3}\left(m_{Z}\right)}{\alpha_{3}\left(M_{\mathrm{SUSY}}\right)}\right]^{\frac{2}{7}}\left[\frac{\alpha_{2}\left(m_{Z}\right)}{\alpha_{2}\left(M_{\mathrm{SUSY}}\right)}\right]^{\frac{27}{38}}\left[\frac{\alpha_{1}\left(m_{Z}\right)}{\alpha_{1}\left(M_{\mathrm{SUSY}}\right)}\right]^{-\frac{23}{82}} .
\end{aligned}
$$

Here, $A_{L}=1.25$ takes care of the long distance renormalization effects coming from QCD [20]. The hadron matrix elements are calculated using lattice techniques in [21] and are found to be

$$
\begin{aligned}
\left\langle\pi^{0}\left|(u d)_{L} u_{R}\right| p\right\rangle_{1} & =\left\langle\pi^{0}\left|(u d)_{R} u_{L}\right| p\right\rangle_{1} \\
& =-0.131(4)(13) \mathrm{GeV}^{2}, \\
\left\langle\pi^{0}\left|(u d)_{L} u_{R}\right| p\right\rangle_{2} & =\left\langle\pi^{0}\left|(u d)_{R} u_{L}\right| p\right\rangle_{2} \\
& =-0.118(3)(12) \mathrm{GeV}^{2} .
\end{aligned}
$$

\section{A. Pure gravity mediation}

In order to calculate the proton lifetime in supersymmetric unification models, we need to specify the SUSY breaking spectrum. Although dimension- 6 proton decay is not strongly dependent on the sparticle spectrum, the proton lifetime does depend on these masses through the running of the gauge couplings. The largest effect to the gauge couplings unification comes from incomplete representations of SU(5). In the MSSM, the relevant particles are the gauginos and the Higgsinos. In models like the constrained MSSM (CMSSM) [22-24], all supersymmetric particles are relatively degenerate. This means that the gauginos and the Higgsinos contribute to the running over roughly the same number of orders of magnitude. In contrast, models like pure gravity mediation (PGM) have loop-suppressed gauginos, due to their anomaly mediation origins $[25,26],{ }^{9}$ compared to the rest of the supersymmetry

\footnotetext{
${ }^{9}$ See Ref. [27] for the path-integral derivation of the anomaly mediated gaugino mass.
} 
breaking spectrum. This means that the gauginos contribute to the running of the gauge couplings over more energy scales than the rest of the SUSY particles. This type of SUSY spectrum flattens the running of $g_{3}$ and steepens the running of $g_{2}$ above the gaugino masses, which pushes up the GUT scale. This will have implications for the proton lifetime. In the following discussion, we focus on this type of the SUSY spectrum, that is a PGM [28-36] spectrum. Product group models of unification tend to have a much too short proton lifetime. As we will see below, a PGM mass spectrum helps push up the unification scale which will have a mild but nontrivial effect on the proton lifetime.

Now, we examine the proton lifetime for the product group unification model found in [9]. We use the SSARD code to evolve the gauge couplings, determine the supersymmetric spectrum, and calculate the proton lifetime [37]. We take a PGM spectrum, which is described below, at the inputs scale defined where $g_{1}=g_{2}$. Since universal pure gravity mediation is rather restrictive, we will broaden our scope and included nonuniversal Higgs masses. This will make it easier to get the correct Higgs mass and allow us to see what kind of proton lifetimes DUNE and Hyper-K should expect. The code evolves the masses and couplings to the weak scale and checks that electroweak symmetry breaking (EWSB) is viable. The Higgs mass is also calculated to verify that it meets experimental constraints.

Before we present our results, we give a short review of pure gravity mediation. In pure gravity mediation, it is assumed that the field breaking supersymmetry is charged. Because of this charge, the gaugino masses are forbidden, since the following operator is forbidden:

$$
W \supset \frac{c_{g} Z}{M_{P}} \mathcal{W W}
$$

where $\mathcal{W}$ is the gauge kinetic function, $Z$ is the SUSY breaking field, and $M_{P}$ is the Planck mass. The leading order contribution to the gaugino masses is then generated by anomaly mediation at the loop level,

$$
M_{i}=\frac{b_{i} g_{i}^{2}}{16 \pi^{2}} m_{3 / 2}
$$

where $b_{i}=33 / 5,1,-3$ for $i=1,2,3$.

The soft masses are a quite different. A charged supersymmetry breaking field, in general, cannot forbid the sfermion mass terms. The sfermions then get a mass of order $m_{3 / 2}$, from operators of the form

$$
K \supset \frac{c_{i j}|Z|^{2}}{M_{P}^{2}} \Phi_{i}^{\dagger} \Phi_{j} .
$$

The theory also has a supersymmetric bilinear mass,

$$
W \supset \mu_{0} H_{u} H_{d}
$$

where $H_{u, d}$ are the up and down Higgs masses, respectively. Because the Higgs fields have an $R$-charge of zero in product group unification models, the above Higgs bilinear term is forbidden. Even if the Higgs fields have zero R-charge, the Higgs bilinear mass terms can be generated in two ways. First, a Giudice-Masiero/Inoue-KawasakiYamaguchi-Yanagida/Casas-Muñoz term [38-40],

$$
\delta K=c_{K} H_{u} H_{d}+\text { H.c. },
$$

will generate an effective Higgs bilinear mass of order $m_{3 / 2}$ as well as supersymmetric breaking $B$-term for the Higgs. The other source of a Higgs bilinear term is from a nonrenormalizable operator of the form

$$
\Delta W=\frac{c_{W}\left\langle W_{h}\right\rangle}{M_{P}^{2}} H_{u} H_{d},
$$

where $W_{h}$ is the Hidden sector superpotential which has a nonzero VEC and is responsible for generating the gravitino mass. Thus, this contribution to the Higgs bilinear mass is again of order $m_{3 / 2}$. If we consider both of these terms, we find that the supersymmetric and supersymmetry breaking Higgs bilinear are linear independent,

$$
\begin{gathered}
\mu=\left(c_{W}+c_{K}\right) m_{3 / 2}, \\
B \mu=\left(-c_{W}+2 c_{K}\right) m_{3 / 2}^{2},
\end{gathered}
$$

where $B$ is the supersymmetry breaking Higgs bilinear mass.

Since the simplest model found in [33] captures all the relevant features of PGM, we will start our examination with universal soft masses at the input scale and then extend our study to include nonuniversal Higgs masses. The free parameters of this theory are

$$
m_{3 / 2} \quad \tan \beta
$$

The Higgs bilinear masses, $\mu$ and $B$, are determined by the electroweak breaking, ${ }^{10}$ leaving just two free parameters. Since this will be rather restrictive, we further examine the case where the Higgs soft masses are free parameters giving the following set of free parameters:

$$
m_{3 / 2} \quad \tan \beta \quad m_{H_{u}}^{2} \quad m_{H_{d}}^{2} .
$$

\section{B. Proton lifetime}

In this section, we present the results of our calculation of the proton lifetime for minimal product group unification discussed above. In Fig. 1, we compare the $g_{1 H}^{2}$ dependence

\footnotetext{
${ }^{10}$ This is equivalent to determining $c_{W, K}$.
} 

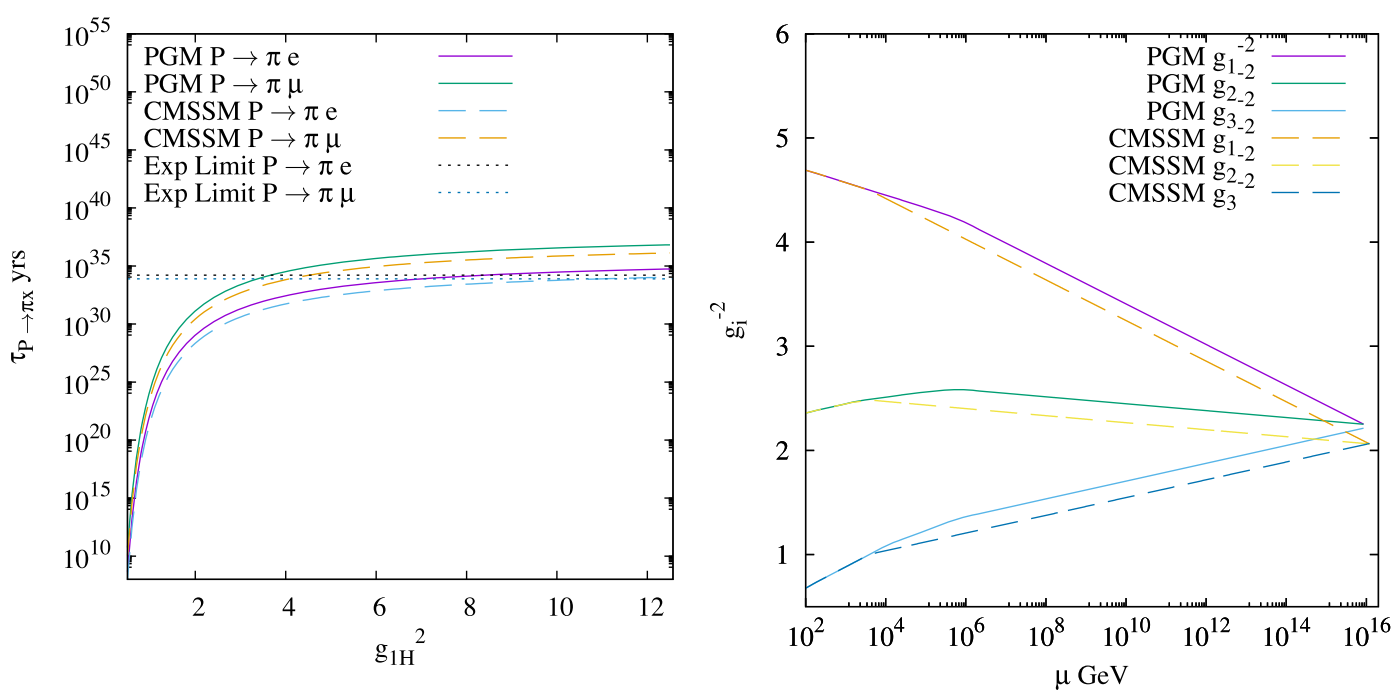

FIG. 1. Left: the proton lifetime as a function of $g_{1 H}^{2}$ at the GUT scale of $\mathcal{O}(\langle Q\rangle)$. The perturbative GUT below the cutoff scale $\Lambda=4 \pi\langle Q\rangle$ is achieved for $g_{1 H}^{2} \leq 3.68$ [see Eq. (14)]. The horizontal dotted lines show the current experimental limits on the proton life time of the modes, $p \rightarrow \pi^{0}+e^{+}$and $p \rightarrow \pi^{0}+\mu^{+}$[15], respectively. Right: the coupling unification for given SUSY spectrum. The better the couplings unify, the lower $M_{X}$ is for a given $g_{1 H}^{2}(\langle Q\rangle)$ [see Eq. (8)].

of the proton lifetime for a pure gravity mediation spectrum with $m_{3 / 2}=700 \mathrm{TeV}, \mu<0$, and $\tan \beta=2.1$ to that for a CMSSM spectrum with $m_{1 / 2}=1.75 \mathrm{TeV}, m_{0}=4 \mathrm{TeV}$, $A_{0} / m_{0}=2, \mu>0$, and $\tan \beta=20$. These values are chosen to obtain a relatively good Higgs mass; however, varying these numbers will not change our conclusions significantly. As is clearly seen, the lifetime is smaller by a nontrivial amount for the CMSSM spectrum. This is ultimately due to the fact that the gauge couplings unify better in the CMSSM, which is also seen in Fig. 1.

Figure 1 also shows the sharp suppression of the proton lifetime near $g_{1 H}^{2}=1$. This is due to the exponential suppression of $M_{X}$ as $g_{1 H}^{2}$ becomes smaller at the GUT scale; see Eq. (8). We see that the proton lifetime is too short unless $g_{1 H}^{2} \gtrsim 6$ even for a PGM spectrum. This contradicts the constraint coming from well-defined Yukawa couplings which requires $g_{1 H}^{2} \lesssim 3.68$ as seen in Eq. (14). Thus, this minimal model of product group unification is ruled out. This leads us to consider nonminimal models of product group unification.

\section{LIGHT COLORED PARTICLES}

\section{A. SU(5) incomplete multiplet below the GUT scale}

As we saw in the previous section, proton decay constraints rule out the simplest product group unification models. The short lifetime of the proton is attributed to the light $X$ bosons, which was a consequence of our separations of the cutoff scale and the GUT scale.

A simple way to address the proton lifetime problem is to add additional representations of SU(5) with the SU(2) and $\mathrm{SU}(3)$ components having different masses. In fact, product unification model can easily achieve the SU(5) incomplete multiplets by introducing an additional $\mathbf{5}, \overline{\mathbf{5}}\left(\Phi^{\prime}, \bar{\Phi}^{\prime}\right.$ in Table I) and 2, $\overline{2}(\Theta, \bar{\Theta}$ in Table I). These fields are coupled to the $\mathrm{SU}(5) \times \mathrm{U}(2)_{\mathrm{H}}$ breaking fields, $Q, \bar{Q}$, in the following way:

$$
\Delta W=\lambda \Phi^{\prime} Q \bar{\Theta}+\lambda \bar{\Phi}^{\prime} \bar{Q} \Theta+\mu_{5} \Phi^{\prime} \bar{\Phi}^{\prime} .
$$

Here, we have taken the Yukawa couplings equal for simplicity. In this model, the doublets of the $\Phi^{\prime}, \bar{\Phi}^{\prime}$ pair up with the $\Theta, \bar{\Theta}$ and obtain mass from the VEV of $Q, \bar{Q}$, while the triplets of $\Phi^{\prime}, \bar{\Phi}^{\prime}$ obtain the mass of $\mu_{5}$. In this way, the SU(5) incomplete multiplets below the GUT scale are achieved without fine-tuning.

If $\mu_{5} \ll \lambda\langle Q\rangle$, the matching conditions in Eqs. (5)-(7) get nontrivial corrections. The expressions for $M_{X}$ is then modified to

$$
M_{X} \simeq\left(\frac{M_{G_{0}}}{\mu_{5}}\right)^{\frac{2}{15}} M_{X_{0}}
$$

where $M_{G_{0}}, M_{X_{0}}$ are the scale the coupling unify at and the heavy gauge boson mass for the case without the additional $\Phi^{\prime}, \bar{\Phi}^{\prime}$, respectively. We have taken $\lambda v=M_{G}$ to maximize the effect of $\Phi^{\prime}, \bar{\Phi}^{\prime}$. Because $M_{X}$ scales vary slowly with $\mu_{5}$ in this expression, we will have to take $\mu_{5} \ll M_{G}$.

Now, we look at the modifications to the RG running of the hidden sector gauge couplings from these additional states. The beta function of $g_{1 H}$ is modified to

$$
\frac{d g_{1 H}^{2}}{d \ln \mu}=7 \frac{g_{1 H}^{4}}{8 \pi^{2}},
$$

giving 


$$
g_{1 H}^{2}(\Lambda / 4 \pi)=3.29
$$

if $g_{1 H}^{2}(\Lambda)=4 \pi$. This slight modification to the upper bound on $g_{1 H}^{2}$ can easily be offset by reducing $\mu_{5}$. As we will see below, this will allow us to get a proton lifetime beyond the current experimental limit. However, because of the slow scaling of $M_{X}$ seen in Eq. (32), the proton lifetime still has an upper limit.

\section{B. Suppressing $\mu_{5}$ with a PQ symmetry}

Before we present our results, we wish to motivate the suppression of $\mu_{5}$ below the GUT scale. The most attractive possibility is the PQ symmetry which solves the strong $C P$ problem $[41,42]$. In the PQ mechanism, $\theta$-angle of QCD is canceled by the VEV of the axion $[43,44]$ associated with the spontaneous breaking of the PQ symmetry.

The PQ mechanism is particularly attractive when its breaking scale is of $10^{10-12} \mathrm{GeV}$ for which the axion is a natural candidate for cold dark matter. For example, if the PQ breaking is broken before inflation, the axion dark matter density is given by the misalignment mechanism,

$$
\Omega_{a} h^{2}=0.18 \theta_{a}^{2}\left(\frac{F_{a}}{10^{12} \mathrm{GeV}}\right)^{1.18}\left(\frac{\Lambda_{\mathrm{QCD}}}{400 \mathrm{MeV}}\right) .
$$

Here, $\theta_{a}$ is the initial misalignment angle of the axion, $F_{a}$ is the PQ breaking scale, and $\Lambda_{\mathrm{QCD}}$ is the QCD scale [45] (see also $[46,47]){ }^{11}$ If the PQ breaking takes place after inflation, on the other hand, cosmic strings are formed at the phase transition of the PQ breaking. The axion winds $N_{\mathrm{w}}(\geq 1)$-times around the cosmic string, and hence, the cosmic string is attached by $N_{\mathrm{w}}$ domain walls when the axion obtains a nontrivial scalar potential due to the QCD effect. For a model with $N_{\mathrm{w}}>1$, the string-wall network is stable and dominates the energy density immediately, which is not consistent with our Universe. For a model with $N_{\mathrm{w}}=1$, the string-wall network is unstable, and it disappears immediately. In this case, the axion dark matter is dominated by the contributions emitted from the decay of the string-wall network [49] (see also [50]),

$\Omega_{a} h^{2}=0.035 \pm 0.012\left(\frac{F_{a}}{10^{10} \mathrm{GeV}}\right)^{1.19}\left(\frac{\Lambda_{\mathrm{QCD}}}{400 \mathrm{MeV}}\right)$.

The PQ-breaking scale is also constrained to be $F_{a} \gtrsim$ $10^{9} \mathrm{GeV}$ from astrophysical phenomena [51-54]. From

\footnotetext{
${ }^{11}$ In this case, the quantum fluctuation of the axion during inflation leads to the isocurvature fluctuation of the axion dark matter density. As its amplitude is proportional to the Hubble parameter during inflation, $H_{I}$, the cosmic microwave background constraints on the isocurvature fluctuation puts a constraint, $H_{I} \lesssim 10^{7-8} \mathrm{GeV}$, when the axion is the dominant dark matter (see, e.g., [48]).
}

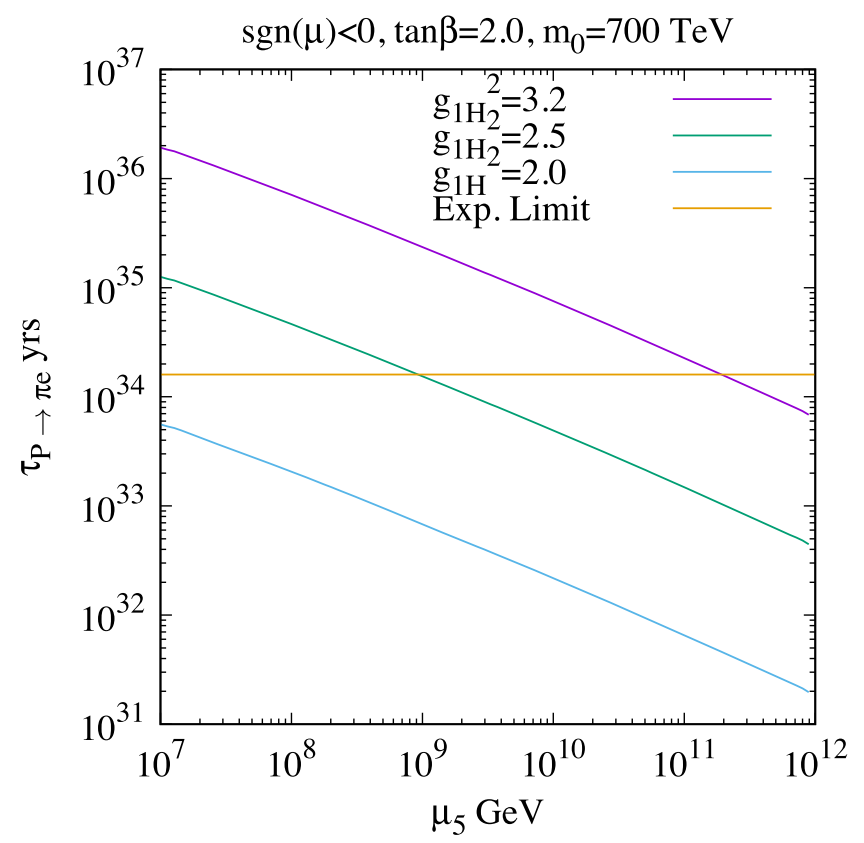

FIG. 2. $\mu_{5}$ dependence of proton lifetime, $p \rightarrow \pi^{0}+e^{+}$in PGM for given value of $g_{1 H}^{2}$ at the GUT scale.

these considerations, we assume the PQ-breaking scale of $10^{10-12} \mathrm{GeV}$ in the following discussion.

To associate $\mu_{5}$ with the PQ-symmetry breaking, we introduce a PQ-symmetry breaking field $P$, in which the axion resides as

$$
P=\frac{F_{a}}{\sqrt{2}} e^{-i a / F_{a}}
$$

The PQ charges can be found in Table I. ${ }^{12}$ As there is only one pair of $\Phi^{\prime}, \bar{\Phi}^{\prime}$, the domain wall number $N_{\mathrm{w}}=1$ in this model. With this PQ charge assignment, the supersymmetric mass term for $\Theta \bar{\Theta}$ is forbidden as is the $\mu_{5}$ term and we instead have

$$
\Delta W=\lambda \Phi^{\prime} Q \bar{\Theta}+\lambda \bar{\Phi}^{\prime} \bar{Q} \Theta+\lambda_{P} P \Phi^{\prime} \bar{\Phi}^{\prime},
$$

with $\lambda$ 's being the coupling constants of order of unity. The effective $\mu_{5}$ is then given by $\mu_{5}=\lambda_{P}\langle P\rangle$. The $\operatorname{VEV}$ of $Q, \bar{Q}$ give masses only to the doublets, while the triplets of $\Phi^{\prime}, \bar{\Phi}^{\prime}$ obtain the much smaller mass, $\mu_{5} \ll \lambda_{P}\langle Q\rangle=\mathcal{O}\left(M_{G}\right)$.

As we will see shortly, this connection of $\mu_{5}$ and the PQ breaking scale will put the proton lifetime in reach of coming proton decay experiments due to Eq. (32). Furthermore, the possibility of axion dark matter makes

\footnotetext{
${ }^{12}$ We set the PQ charge of $\bar{\Phi}^{\prime}$ vanishing, which allows slight mixing between $\bar{\Phi}$ and $\bar{\Phi}^{\prime}$ through which the triplets in $\Phi^{\prime}$ and $\bar{\Phi}^{\prime}$ can decay into the MSSM fields. A sizable mixing between $\bar{\Phi}$ and $\bar{\Phi}^{\prime}$ affects the proton lifetime and the branching fractions [55].
} 
the parameter space of models like pure gravity mediation much less restricted, as we will see below.

Before closing this section, let us comment on the axion coupling to photons through the electromagnetic anomaly,

$$
\mathcal{L}=\frac{1}{4} G_{a \gamma \gamma} a F \tilde{F}
$$

where $F$ and $\tilde{F}$ denote the QED field strength and its dual. The coupling constant $G_{a \gamma \gamma}$ is given by

$$
G_{a \gamma \gamma}=\frac{\alpha}{2 \pi}\left(c_{a \gamma \gamma}-\frac{2}{3} \frac{1+4 z}{1+z}\right) \frac{1+z}{z^{1 / 2}} \frac{m_{\pi}}{m_{a}} \frac{1}{f_{\pi}},
$$

where $f_{\pi} \simeq 92 \mathrm{MeV}, z=m_{u} / m_{d} \simeq 0.553 \pm 0.043$ [56]. Here we have inserted the axion mass,

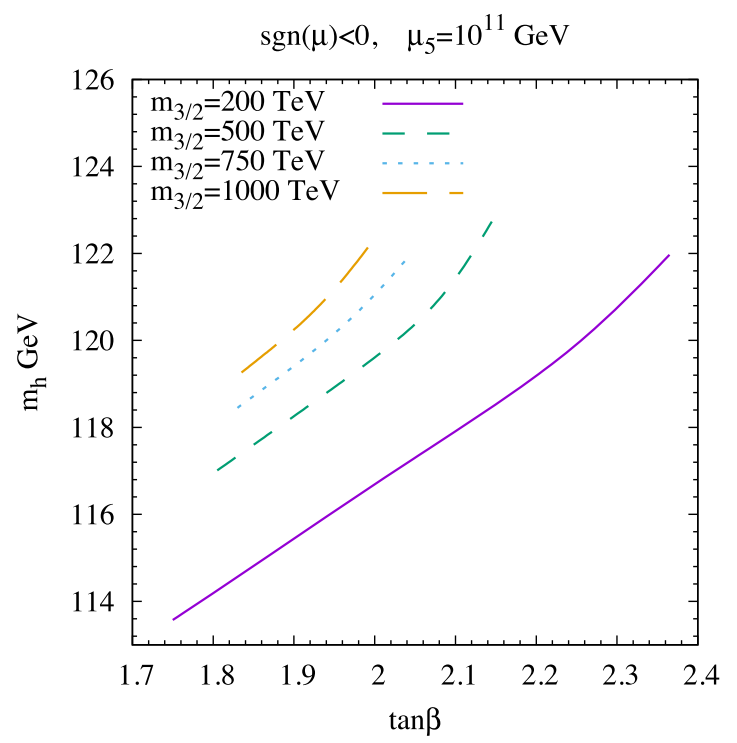

$$
m_{a}=\frac{z^{1 / 2}}{1+z} \frac{f_{\pi}}{F_{a}} m_{\pi}
$$

In the present model, $c_{a \gamma \gamma}$ is given by $c_{a \gamma \gamma}=2 / 3$, which should be compared with the complete GUT KSVZ multiplet of $\mathbf{5}, \overline{\mathbf{5}}$ giving $c_{a \gamma \gamma}=8 / 3$ (see, e.g., [57]). As a result, the present model predicts an axion coupling to QED which is 3 times larger, for a given axion mass, than in the conventional GUT model with a complete KSVZ multiplet.

\section{RESULTS}

Here we show the results of our study of product group unification with the addition of a pair of light triplet quarks. We begin with universal pure gravity mediation and study the effect of $\mu_{5}$ on the lifetime of the proton. In Fig. 2, we plot the proton lifetime versus $\mu_{5}$ for different values of $g_{1 H}^{2}$

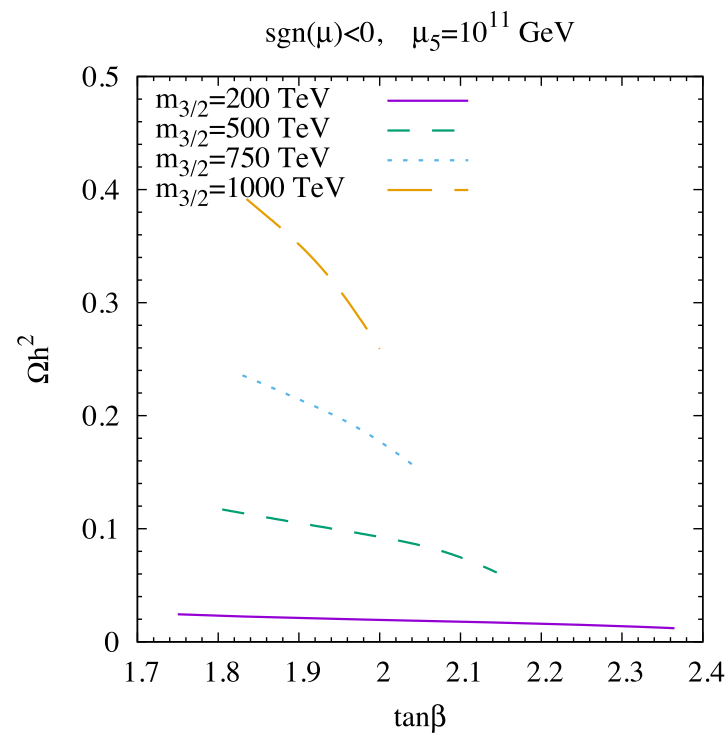

$\operatorname{sgn}(\mu)<0, \quad \mu_{5}=10^{11} \mathrm{GeV}$

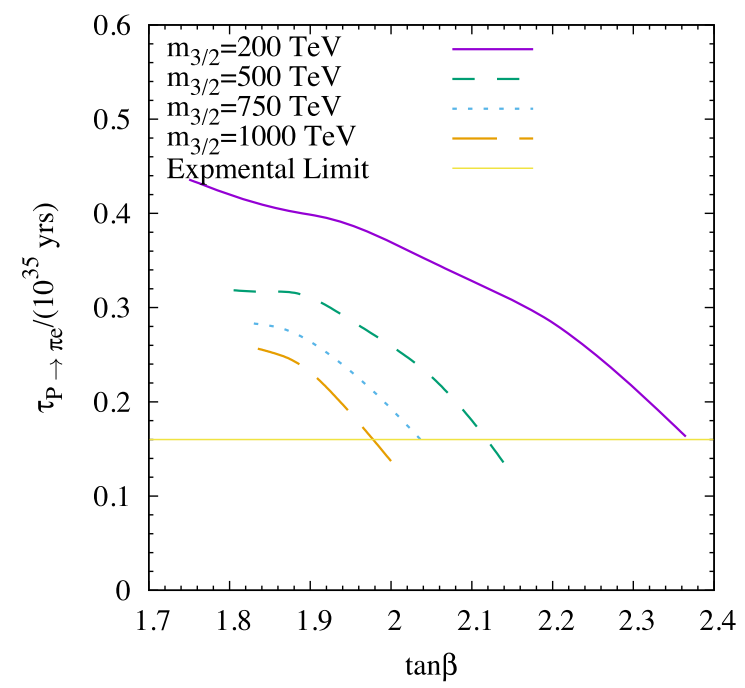

FIG. 3. The lightest Higgs boson mass, $m_{h}$, the LSP abundance, and the proton lifetime for the universal PGM as a function of tan $\beta$. 
for a PGM spectrum with $m_{3 / 2}=700 \mathrm{TeV}, \tan \beta=2$, and $\mu>0$. As can be seen in this figure, the largest lifetime occurs for larger values of $g_{1 H}^{2}$ and smaller values of $\mu_{5}$. However, the separation of strong dynamics from the GUT scale limits how large we can take $g_{1 H}^{2}$, Eq. (34). Because of our naive estimation for $g_{1 H}^{2}$ in Eq. (34), we take $g_{H_{1}}^{2}=3.2$ as the maximal value of $g_{1 H}^{2}$ in Fig. 2 using it only as a guide. However, as can be estimated from the figure, a small change in $g_{1 H}^{2}$ does not affect our conclusions too much. Furthermore, from Fig. 2, it is clear that the lifetime scales quite close to our estimate in Eq. (32) and it is quite difficult to push $\mu_{5}$ beyond $10^{12} \mathrm{GeV}$.

Next, we consider universal PGM with the addition of $\Phi^{\prime}, \bar{\Phi}^{\prime}$. Our results can be seen in Fig. 3. The lines terminate at larger $\tan \beta$ due to EWSB failing, that is, equations need $|\mu|^{2}<0$. For smaller $\tan \beta$, the lines terminate due to a nonperturbative Yukawa coupling. Although the edge with larger $\tan \beta$ may be allowed due to the large errors in calculating the Higgs mass, much of the parameter space is still ruled out by the Higgs mass measurement, $m_{h}=$ $125.10 \pm 0.14 \mathrm{GeV}$ [58]. With $\mu_{5}=10^{11} \mathrm{GeV}$, the proton lifetime is sufficiently long for most of the parameter space. However, for the edge with larger $\tan \beta$, where the Higgs mass is most consistent with the measured value, the proton lifetime tends to be too short. This means universal PGM needs $\mu_{5}<10^{11} \mathrm{GeV}$. In the figure, we also show the thermal relic lightest supersymmetric particle (LSP) contribution to the dark matter abundance. The LSP is mostly Wino-like neutralino in the PGM spectrum. For $m_{3 / 2} \lesssim 500 \mathrm{TeV}$, the Wino dark matter density is insufficient to explain the measured value. However, for this range
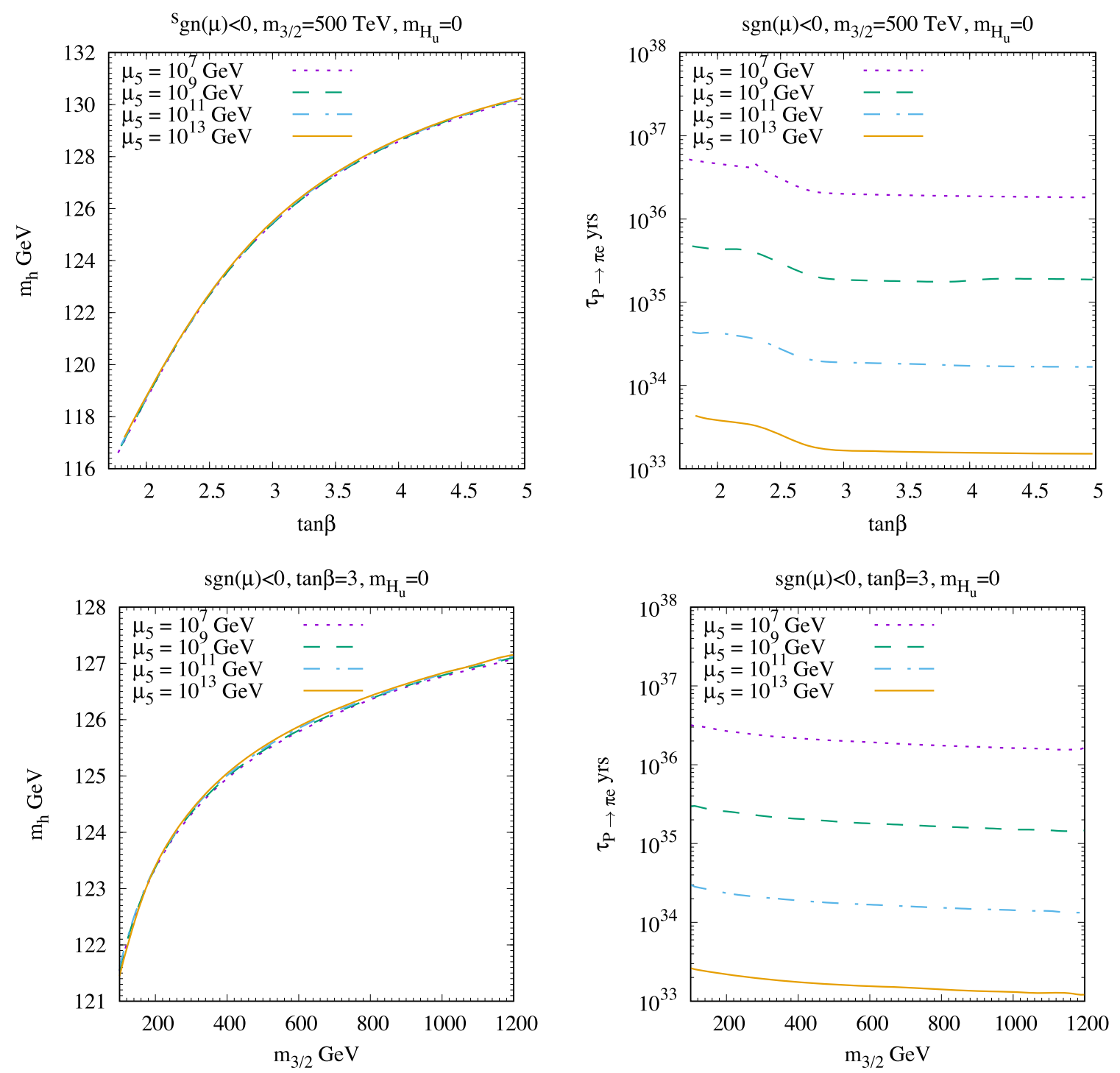

FIG. 4. The $\tan \beta$ (top) and the $m_{3 / 2}$ (bottom) dependence of $m_{h}$ (left) and of the proton lifetime (right). Here, we take $m_{H_{u}}^{2}=m_{3 / 2}^{2}$ and $m_{H_{d}}^{2}=0$ as an example of the nonuniversal Higgs mass. 
of $m_{3 / 2}$ and value of $\mu_{5}$, the axion can make up a large fraction of the dark matter.

Since it is very possible that the soft masses of PGM are nonuniversal, we will look at more generic mass spectra. Since the dimension- 6 proton decay is quite insensitive to the sfermion masses, we will consider the case where only the Higgs soft masses are nonuniversal. This will relax the tension on the proton lifetime coming from the Higgs mass measurement and allow us to more fully explore the parameter space consistent with product group unification.

We begin our study of nonuniversal Higgs masses by looking at the dependence of the Higgs boson mass and proton lifetime on $m_{3 / 2}$ and $\tan \beta$ for different values of $\mu_{5}$. To demonstrate the nonuniversal Higgs masses, we take $m_{H_{d}}^{2}=m_{3 / 2}^{2}$ while $m_{H_{u}}^{2}=0$. With the nonuniversal Higgs mass, the successful EWSB is achieved even for $\tan \beta \gtrsim 2$. As is clear from Fig. 4, the lightest Higgs mass strongly depends on $\tan \beta$ and $m_{3 / 2}$ but vary mildly with $\mu_{5}$. This is because the only effect of $\mu_{5}$ is to mildly change the running of the gauge couplings. However, since the couplings are fixed by experiment at the low scale, this effect is quite mild. The proton lifetime, on the other hand, depends quite mildly on both $\tan \beta$ and $m_{3 / 2}$ and very strongly on $\mu_{5}$. This is due to the fact that $\mu_{5}$ can have a significant effect on the running of the gauge couplings for scales above $\mu_{5} \cdot \tan \beta$ and $m_{3 / 2}$, in contrast, only affect the gauge coupling running indirectly through the Higgsino's and gaugino's masses.

The effects discussed above can be seen in Fig. 4. In the top two figures, we see the typical strong dependence of the lightest Higgs mass dependence on $\tan \beta$. The current experimental limit, $m_{h}=125.10 \pm 0.14 \mathrm{GeV}$ [58], combined with the theoretical uncertainties, constrains $\tan \beta$ to be roughly in the range $2.5-4$. The proton lifetime is, as expected, quite mildly dependent on $\tan \beta$ and saturates at about $\tan \beta \simeq 3$. This is due to a saturation of the Higgsino and Wino masses $\tan \beta$ dependence. ${ }^{13}$

For the bottom two figures in Fig. 4, we see the expected strong dependence of the lightest Higgs boson mass on $m_{3 / 2}$, which determines all the sfermion masses. Due to the theoretical uncertainties in Higgs mass calculation, all plotted values are consistent with the measure Higgs boson mass. The proton lifetimes dependence on $m_{3 / 2}$ is through the Higgsino and Wino. The Wino mass is generated through anomaly mediation with a nontrivial threshold correction coming from the Higgsinos and heavy Higgs. Since the Higgsino mass also scales with $m_{3 / 2}$, it is roughly set by the stop mass. However, since this mass dependence

\footnotetext{
${ }^{13}$ The Higgsino's $\tan \beta$ dependence is through the electroweak symmetry breaking conditions, which are how the Higgsino mass, $\mu$, is determined. The Wino gets a $\tan \beta$ dependence through a relatively large threshold correction generated when the Higgsino and heavy Higgs bosons are integrated out.
}

of the gauge couplings is only logarithmic, we only see a mild dependence of the proton lifetime on $m_{3 / 2}$ in Fig. 4.

Next, we look at the $\tan \beta$ versus $m_{3 / 2}$ plane for $m_{H_{u}}^{2}=0$. We choose $m_{H_{u}}^{2}=0$ for simplicity. However, most other values where $m_{H_{u}}^{2}$ is smaller than $m_{3 / 2}^{2}$ by a nontrivial amount would work. The advantage of taking $m_{H_{u}}^{2}<m_{3 / 2}^{2}$ is it restores the freedom in $\tan \beta$. In universal PGM, small values of $\tan \beta$ are needed so that the top Yukawa couplings are large. If the top Yukawa couplings are not large, the radiative corrections to the Higgs soft masses are not large enough to generate radiative EWSB. In Fig. 5, we show the plane of $\tan \beta$ versus $m_{3 / 2}$. The blue dashed line is where the Wino masses give the correct thermal relic density for dark matter. Below this line, the dark matter density is less than the experimentally measured value. In the region below this line, the dark matter can be a mixture of the Wino and axion. As can be seen from the green short dashed line, the proton lifetime, which is labeled in units of $10^{35}$ years, is quite small in the regions which is preferred by the Higgs mass measurements. This means future experiments will be able to completely rule out all parameter space shown in this figure. Furthermore, the constraints on the proton lifetime push us toward smaller $m_{3 / 2}$ and thus a larger fraction of axion dark matter. Effectively, this model correlates the axion dark matter fraction with proton lifetime.

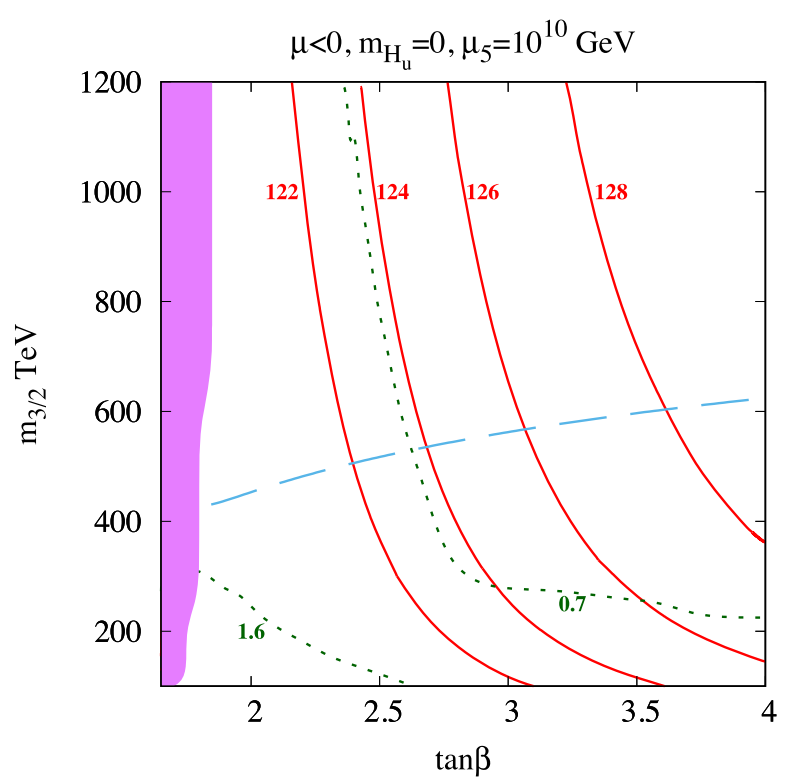

FIG. 5. The $\tan \beta$ and $m_{3 / 2}$ dependence of the lightest Higgs boson mass in units of $\mathrm{GeV}$ (red lines) and the proton lifetime in units of $10^{35}$ years (green dotted lines). Here, we take $m_{H_{u}}^{2}=0$ and $m_{H_{d}}^{2}=m_{3 / 2}^{2}$ as an example of the nonuniversal Higgs mass. The blue dashed line is where the Wino masses give the correct thermal relic density for dark matter. The pink shaded region is excluded by the nonperturbative Yukawa coupling. 

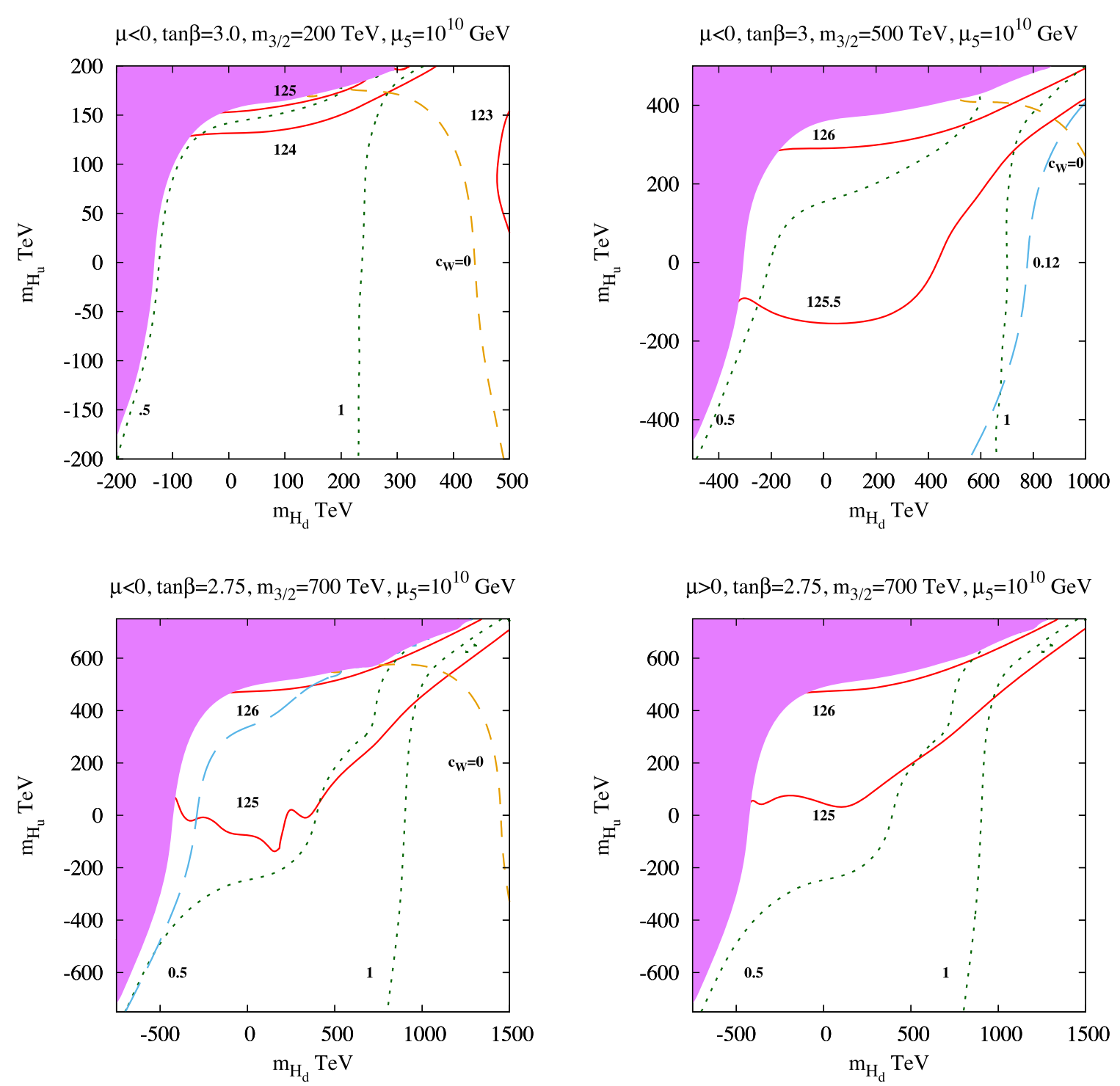

FIG. 6. The $m_{H_{u}}^{2}$ and $m_{H_{d}}^{2}$ dependence of the lightest Higgs boson mass in units of GeV (red lines) and the proton lifetime in units of $10^{35}$ years (green dotted lines). The blue dashed line is where the Wino masses give the correct thermal relic density for dark matter. The yellow dashed line is where $c_{W}=0$. The pink shaded region is excluded by the failure of the EWSB.

The last set of figures, Fig. 6, is for the $m_{H_{d}}$ versus $m_{H_{u}}$ plane. ${ }^{14}$ In these figures, we show how the proton lifetime depends on the Higgs soft masses. In the top left figure, we take $m_{3 / 2}=200 \mathrm{TeV}$. The red contours are the Higgs mass, the green short dotted lines are the proton lifetime in units of $10^{35}$ years, and the yellow short dashed line is where $c_{W}=0$. There is no line corresponding to a dark matter density of 0.12 , since the entire plane has a Wino masses which is too small to give a thermal dark matter density of 0.12 . The proton lifetime varies quite slowly across the entire plane. In fact, most of the plane is within

\footnotetext{
${ }^{14}$ Here, $m_{H_{d}}$ and $m_{H_{u}}$ denote $\operatorname{sign}\left(m_{H_{d}}\right)\left|m_{H_{d}}^{2}\right|{ }^{1 / 2}$ and $\operatorname{sign}\left(m_{H_{u}}\right)\left|m_{H_{u}}^{2}\right|^{1 / 2}$, respectively.
}

reach of upcoming proton decay searches. The pink region along the top and left edge is excluded because the radiative EWSB conditions cannot be met. In the top right figure, we take the same set of parameters except now we take $m_{3 / 2}=500 \mathrm{TeV}$. The lines are the same as the left figure except now we have a blue long dashed line corresponding to the measure relic density 0.12 . Again, the proton lifetime varies slowly and much of the plane is within the reach of upcoming experiments. In the bottom left figure, we take $m_{3 / 2}=700 \mathrm{TeV}$. This figure is similar to the top two figures, except now the dark matter density is too larger over much of the plane. This mean if the Universe follows a standard cosmology, we are constrained to live along the edge of the region where electroweak symmetry breaking fails. The bottom right figure is the same as the bottom left 
except it has $\mu<0$. This drastically affects the dark matter density, since it flips the sign of the threshold correction to the Wino coming from integrating out the Higgsino. This drastically reduced the dark matter density and makes the entire plane have a dark matter density less than 0.12 .

\section{CONCLUSIONS}

In this paper, we discussed the proton decay for the simplest product group unification based on $\mathrm{SU}(5) \times \mathrm{U}(2)_{\mathrm{H}}$. The product group unification is attractive alternative which solves the doublet-triplet splitting problem and the dimension-5 proton decay problem by $\mathrm{R}-$ symmetry. By requiring the model be perturbative up to the cutoff scale, we find that the effective GUT scale is considerably smaller than the conventional GUT scale, which roughly corresponds to the scale the MSSM gauge coupling constants unify. As a result, we find that the minimal setup of the $\mathrm{SU}(5) \times \mathrm{U}(2)_{\mathrm{H}}$ model has been excluded by the proton decay experiments.

We also showed that a simple extension of the model with SU(5) incomplete multiplets can rectify this problem. It should be noted that the incomplete multiplets can be achieved in product group unification without fine-tuning. Although the proton lifetime does not depend on the MSSM spectrum significantly, we demonstrated the parameter dependence by taking the PGM spectrum as an example. As a result, we found that the proton lifetime in the extended model is in reach of coming experiments like DUNE and Hyper-K, when the mass of the incomplete multiplet is associated with the Peccei-Quinn symmetry. The dark matter in this model consists of an admixture of the Wino LSP and the axion. The axion coupling to QED is enhanced by a factor of 3 compared with the KSVZ axion model with a GUT complete $\mathbf{5}, \overline{\mathbf{5}}$ multiplet. Therefore, this scenario can be tested by combining the proton decay searches, the LSP (Wino) searches and the axion searches.

Product group unification models based on $\mathrm{SU}(5) \times$ $\mathrm{U}(3)_{\mathrm{H}}$ are also possible [9-12,14]. The minimal model is likewise ruled out due to a short dimension-6 proton decay lifetime. Similar to what was done in here, this class of models can be salvaged by the addition of intermediate scale SU(2) doublet fields. However, there is no strong motivation for these intermediate mass doublets. Unlike the $\mathrm{SU}(5) \times \mathrm{U}(2)_{\mathrm{H}}$ case, where the intermediate scale mass of the colored triplets is set by PQ breaking scale. Thus, the preferred product group unification models is the one we have considered based on $\mathrm{SU}(5) \times \mathrm{U}(2)_{\mathrm{H}}$.

\section{ACKNOWLEDGMENTS}

This work was supported by Grant-in-Aid for Scientific Research from the Ministry of Education, Culture, Sports, Science, and Technology (MEXT), Japan, Grants No. 17 H02878 (M. I. and T. T. Y.), No. 18H05542 (M. I.), No. $19 \mathrm{H} 05810$ (T. T. Y.), and by World Premier International Research Center Initiative (WPI), MEXT, Japan. J. L. E. and T. T. Y. would like to thank IPMU for their hospitality during the completion of this work.
[1] N. Sakai and T. Yanagida, Nucl. Phys. B197, 533 (1982).

[2] S. Weinberg, Phys. Rev. D 26, 287 (1982).

[3] J. Ellis, J. L. Evans, A. Mustafayev, N. Nagata, and K. A. Olive, Eur. Phys. J. C 76, 592 (2016).

[4] J. Ellis, J. L. Evans, N. Nagata, D. V. Nanopoulos, and K. A. Olive, Eur. Phys. J. C 77, 232 (2017).

[5] J. Ellis, J. L. Evans, N. Nagata, K. A. Olive, and L. VelascoSevilla, Eur. Phys. J. C 80, 332 (2020).

[6] A. Masiero, D. V. Nanopoulos, K. Tamvakis, and T. Yanagida, Phys. Lett. B 115, 380 (1982).

[7] B. Grinstein, Nucl. Phys. B206, 387 (1982).

[8] K. Hamaguchi, S. Hor, and N. Nagata, J. High Energy Phys. 11 (2020) 140.

[9] M. Ibe and T. Watari, Phys. Rev. D 67, 114021 (2003).

[10] K. Izawa and T. Yanagida, Prog. Theor. Phys. 97, 913 (1997).

[11] T. Yanagida, Phys. Lett. B 344, 211 (1995).

[12] J. Hisano and T. Yanagida, Mod. Phys. Lett. A 10, 3097 (1995).

[13] T. Hotta, K. Izawa, and T. Yanagida, Phys. Rev. D 54, 6970 (1996).
[14] T. Hotta, K.-I. Izawa, and T. Yanagida, Prog. Theor. Phys. 95, 949 (1996).

[15] K. Abe et al. (Super-Kamiokande Collaboration), Phys. Rev. D 95, 012004 (2017).

[16] J. E. Kim, Phys. Rev. Lett. 43, 103 (1979).

[17] M. A. Shifman, A. Vainshtein, and V. I. Zakharov, Nucl. Phys. B166, 493 (1980).

[18] B. Abi et al. (DUNE Collaboration), arXiv:1807.10334.

[19] K. Abe et al. (Hyper-Kamiokande Collaboration), arXiv: 1805.04163.

[20] T. Nihei and J. Arafune, Prog. Theor. Phys. 93, 665 (1995).

[21] Y. Aoki, T. Izubuchi, E. Shintani, and A. Soni, Phys. Rev. D 96, 014506 (2017).

[22] M. Drees and M. M. Nojiri, Phys. Rev. D 47, 376 (1993); H. Baer and M. Brhlik, Phys. Rev. D 53, 597 (1996); 57, 567 (1998); H. Baer, M. Brhlik, M. A. Diaz, J. Ferrandis, P. Mercadante, P. Quintana, and X. Tata, Phys. Rev. D 63, 015007 (2000); J. R. Ellis, T. Falk, G. Ganis, K. A. Olive, and M. Srednicki, Phys. Lett. B 510, 236 (2001).

[23] G. L. Kane, C. F. Kolda, L. Roszkowski, and J. D. Wells, Phys. Rev. D 49, 6173 (1994); J. R. Ellis, T. Falk, 
K. A. Olive, and M. Schmitt, Phys. Lett. B 388, 97 (1996); V. D. Barger and C. Kao, Phys. Rev. D 57, 3131 (1998); J. R. Ellis, T. Falk, K. A. Olive, and M. Schmitt, Phys. Lett. B 413, 355 (1997); J. R. Ellis, T. Falk, G. Ganis, K. A. Olive, and M. Schmitt, Phys. Rev. D 58, 095002 (1998); J. R. Ellis, T. Falk, G. Ganis, and K. A. Olive, Phys. Rev. D 62, 075010 (2000); L. Roszkowski, R. Ruiz de Austri, and T. Nihei, J. High Energy Phys. 08 (2001) 024; A. Djouadi, M. Drees, and J. Kneur, J. High Energy Phys. 08 (2001) 055; J. R. Ellis, K. A. Olive, and Y. Santoso, New J. Phys. 4, 32 (2002); H. Baer, C. Balazs, A. Belyaev, J. K. Mizukoshi, X. Tata, and Y. Wang, J. High Energy Phys. 07 (2002) 050; J. R. Ellis, K. A. Olive, Y. Santoso, and V. C. Spanos, Phys. Lett. B 565, 176 (2003); H. Baer and C. Balazs, J. Cosmol. Astropart. Phys. 05 (2003) 006; A. Lahanas and D. V. Nanopoulos, Phys. Lett. B 568, 55 (2003); U. Chattopadhyay, A. Corsetti, and P. Nath, Phys. Rev. D 68, 035005 (2003); C. Munoz, Int. J. Mod. Phys. A 19, 3093 (2004); R. L. Arnowitt, B. Dutta, and B. Hu, in 4th International Conference on Physics Beyond the Standard Model: Beyond the Desert (BEYOND 03) (Cambridge University Press, Tegernsee, 2003), pp. 25-41; J. Ellis and K. A. Olive, in Supersymmetric Dark Matter Candidates (Cambridge University Press, Tegernsee, 2010), pp. 142163; J. Ellis and K. A. Olive, Eur. Phys. J. C 72, 2005 (2012); O. Buchmueller et al., Eur. Phys. J. C 74, 2809 (2014).

[24] J. Ellis, F. Luo, K. A. Olive, and P. Sandick, Eur. Phys. J. C 73, 2403 (2013).

[25] L. Randall and R. Sundrum, Nucl. Phys. B557, 79 (1999).

[26] G. F. Giudice, M. A. Luty, H. Murayama, and R. Rattazzi, J. High Energy Phys. 12 (1998) 027.

[27] K. Harigaya and M. Ibe, Phys. Rev. D 90, 085028 (2014).

[28] M. Ibe, T. Moroi, and T. Yanagida, Phys. Lett. B 644, 355 (2007).

[29] M. Ibe and T. T. Yanagida, Phys. Lett. B 709, 374 (2012).

[30] M. Ibe, S. Matsumoto, and T. T. Yanagida, Phys. Rev. D 85, 095011 (2012).

[31] B. Bhattacherjee, B. Feldstein, M. Ibe, S. Matsumoto, and T. T. Yanagida, Phys. Rev. D 87, 015028 (2013).

[32] N. Arkani-Hamed, A. Gupta, D. E. Kaplan, N. Weiner, and T. Zorawski, arXiv:1212.6971.

[33] J. L. Evans, M. Ibe, K. A. Olive, and T. T. Yanagida, Eur. Phys. J. C 73, 2468 (2013).
[34] J. L. Evans, K. A. Olive, M. Ibe, and T. T. Yanagida, Eur. Phys. J. C 73, 2611 (2013).

[35] J. L. Evans and K. A. Olive, Phys. Rev. D 90, 115020 (2014).

[36] J. L. Evans, M. Ibe, K. A. Olive, and T. T. Yanagida, Phys. Rev. D 91, 055008 (2015).

[37] Information about this code is available from K. A. Olive: It contains important contributions from J. Evans, T. Falk, A. Ferstl, G. Ganis, F. Luo, A. Mustafayev, J. McDonald, K. A. Olive, P. Sandick, Y. Santoso, V. Spanos, M. Srednicki, and J. Zheng.

[38] G. Giudice and A. Masiero, Phys. Lett. B 206, 480 (1988).

[39] K. Inoue, M. Kawasaki, M. Yamaguchi, and T. Yanagida, Phys. Rev. D 45, 328 (1992).

[40] J. Casas and C. Munoz, Phys. Lett. B 306, 288 (1993).

[41] R. Peccei and H. R. Quinn, Phys. Rev. Lett. 38, 1440 (1977).

[42] R. Peccei and H. R. Quinn, Phys. Rev. D 16, 1791 (1977).

[43] S. Weinberg, Phys. Rev. Lett. 40, 223 (1978).

[44] F. Wilczek, Phys. Rev. Lett. 40, 279 (1978).

[45] M. S. Turner, Phys. Rev. D 33, 889 (1986).

[46] S. Borsanyi et al., Nature (London) 539, 69 (2016).

[47] G. Ballesteros, J. Redondo, A. Ringwald, and C. Tamarit, J. Cosmol. Astropart. Phys. 08 (2017) 001.

[48] M. Kawasaki and K. Nakayama, Annu. Rev. Nucl. Part. Sci. 63, 69 (2013).

[49] T. Hiramatsu, M. Kawasaki, K. Saikawa, and T. Sekiguchi, Phys. Rev. D 85, 105020 (2012); 86, 089902(E) (2012).

[50] A. Ringwald, in Proceedings of the 53rd Rencontres de Moriond on Cosmology (La Thuile, 2018), pp. 241-244.

[51] G. G. Raffelt, Lect. Notes Phys. 741, 51 (2008).

[52] J. H. Chang, R. Essig, and S. D. McDermott, J. High Energy Phys. 09 (2018) 051.

[53] I. G. Irastorza and J. Redondo, Prog. Part. Nucl. Phys. 102, 89 (2018).

[54] K. Hamaguchi, N. Nagata, K. Yanagi, and J. Zheng, Phys. Rev. D 98, 103015 (2018).

[55] M. Ibe, S. Shirai, M. Suzuki, and T. T. Yanagida, Phys. Rev. D 100, 055024 (2019).

[56] H. Leutwyler, Phys. Lett. B 378, 313 (1996).

[57] G. G. di Cortona, E. Hardy, J. P. Vega, and G. Villadoro, J. High Energy Phys. 01 (2016) 034.

[58] P. Zyla et al. (Particle Data Group), Prog. Theor. Exp. Phys. 083C01 (2020). 